\title{
Results of the arterial switch operation for Taussig-Bing variants in the setting of a lower-middle income country: a single institution experience
}

\author{
Mai Tuan Nguyen ${ }^{1}$, Nguyen Ly Thinh Truong ${ }^{1}$, Vinh Quang Tran ${ }^{1}$, Duyen Dinh Mai ${ }^{1}$, and \\ Anh Vuong Doan ${ }^{1}$ \\ ${ }^{1}$ Department of Cardiovascular Surgery Children Heart Center National Children's \\ Hospital Hanoi Vietnam
}

December 6, 2021

\begin{abstract}
Background: This study was conducted to evaluate the surgical results of the arterial switch operation for Taussig-Bing variants, at a single institution in a lower-middle income country. Methods: Between June 2010 and December 2018, all consecutive patients diagnosed with Taussig-Bing variants who underwent the arterial switch operation and ventricular septal defect closure were included in the study. Results: A total of 72 patients of Taussig-Bing variants who underwent arterial switch operation and ventricular septal defect closure. There were 10 early deaths (13.9\%) and 2 late deaths (2.8\%). Intraoperative ventricular septal defect enlargement [hazard ratio (HR) 7.23, 95\% confidence interval (CI) 3.1294-16.7167; $P<0.001$ ], secondary aortic cross clamping (HR 28.38, 95\% CI 4.8427-166.3484; $P<0.001$ ), post-operative pneumonia (HR 5.64, 95\% CI 1.2724-24.9917; $P=0.023$ ), and post-operative sepsis (HR 5.28, 95\% CI 1.3512-20.6553; p = 0.017) were risk factors for overall mortality by competing risk analysis. Sixty patients $(83.3 \%)$ required septoparietal trabeculation division/resection during the arterial switch operation in an attempt to avoid right ventricular outflow tract obstruction. The reoperation rate for right ventricular outflow tract obstruction at last follow up was $6 \%$ (3 patients). The estimated freedom from reoperation for right ventricular outflow tract obstruction at 1 year, 5 year and 9 year was $98.3 \%, 91.9 \%$ and 91.9 , respectively. Conclusions: The results of arterial switch operation for Taussig-Bing variants were satisfactory in the operative setting of a lower-middle income country, and performing extensive septoparietal trabeculation division might reduce the reintervention rate for right ventricular outflow tract obstruction in these patients.
\end{abstract}

Results of the arterial switch operation for Taussig-Bing variants in the setting of a lowermiddle income country: a single institution experience

Mai Tuan Nguyen $\mathrm{MD}^{1}$, Truong Ly Thinh Nguyen $\mathrm{MD}, \mathrm{PhD}^{1}$, Vinh Quang Tran $\mathrm{MD}^{1}$, Duyen Dinh Mai $\mathrm{MD}^{1}$, Anh Vuong Doan $\mathrm{MD}^{1}$

1: Department of Cardiovascular Surgery, Children Heart Center, National Children's Hospital, Hanoi, Vietnam

\section{Short running title: Taussig-Bing variants}

\section{Corresponding author}

Truong Nguyen Ly Thinh, Department of Cardiovascular Surgery, Children Heart Center, National Children's Hospital, 18/879 La Thanh, Dong Da, Hanoi, Vietnam.

Tel.: (+84) 989999001. E-mail: nlttruong@gmail.com 
Funding: The author(s) received no financial support for the research, authorship, and/or publication of this article

\section{Meeting presentation}

Presented at the 34th Annual Meeting of the European Association for Cardio-Thoracic Surgery, Barcelona, Spain, October 3-5, 2020

Word count : 4474 words

\section{Abstract}

Background: This study was conducted to evaluate the surgical results of the arterial switch operation for Taussig-Bing variants, at a single institution in a lower-middle income country.

Methods: Between June 2010 and December 2018, all consecutive patients diagnosed with Taussig-Bing variants who underwent the arterial switch operation and ventricular septal defect closure were included in the study.

Results: A total of 72 patients of Taussig-Bing variants who underwent arterial switch operation and ventricular septal defect closure. There were 10 early deaths $(13.9 \%)$ and 2 late deaths $(2.8 \%)$. Intraoperative ventricular septal defect enlargement [hazard ratio (HR) 7.23, 95\% confidence interval (CI) 3.1294-16.7167; $P<0.001$ ], secondary aortic cross clamping (HR 28.38, 95\% CI 4.8427-166.3484; $P<0.001$ ), post-operative pneumonia (HR 5.64, 95\% CI 1.2724-24.9917; $P=0.023$ ), and post-operative sepsis (HR 5.28, 95\% CI $1.3512-20.6553 ; \mathrm{p}=0.017)$ were risk factors for overall mortality by competing risk analysis. Sixty patients $(83.3 \%)$ required septoparietal trabeculation division/resection during the arterial switch operation in an attempt to avoid right ventricular outflow tract obstruction. The reoperation rate for right ventricular outflow tract obstruction at last follow up was $6 \%$ (3 patients). The estimated freedom from reoperation for right ventricular outflow tract obstruction at 1 year, 5 year and 9 year was $98.3 \%, 91.9 \%$ and 91.9 , respectively.

Conclusions: The results of arterial switch operation for Taussig-Bing variants were satisfactory in the operative setting of a lower-middle income country, and performing extensive septoparietal trabeculation division might reduce the reintervention rate for right ventricular outflow tract obstruction in these patients.

Keywords: Taussig-Bing variants, arterial switch operation, right ventricular outflow tract obstruction

Abstract word count: 253 words

\section{Abbreviations}

ASO: arterial switch operation

VSD: ventricular septal defect

RVOT: right ventricular outflow tract

RVOTO: right ventricular outflow tract obstruction

POD: postoperative day

PA: pulmonary artery

PV: pulmonary valve

Ao: aorta

SPT: septoparietal trabeculation

LMIC: lower-middle income country

\section{Introduction}


The arterial switch operation (ASO) with ventricular septal defect closure (VSD closure) has become the procedure of choice for Taussig-Bing variants (TBV) repair $(1,2)$. Additionally, single-stage total correction might provide better outcomes for these patients with aortic arch hypoplasia or coarctation of the aorta (Ao) (3-5). In the literatures, the short-term and midterm survival outcomes of these patients after the surgical repair of this complex congenital heart disease (CHD) have been excellent $(5,6)$.

However, TBV patients have a high rate of reoperation or reintervention after the ASO, especially related to the development of right ventricular outflow tract obstruction (RVOTO) $(7,8)$. The risk factors for RVOTO after ASO are side-by-side great arteries, aortic arch obstruction, coronary artery anomalies, preoperative presence of subaortic obstruction, and preoperative diagnosis of TBV $(8,9)$.

In this study, we investigated the surgical outcome and risk factors for mortality after the ASO for TBV patients at a single institution from a lower-middle income country (LMIC), and in particular describe our surgical techniques related to RVOT reconstruction by prophylactic resection of the septoparietal trabeculations (SPT), and evaluation of RVOT during follow-up.

\section{Materials and methods}

This study was approved by the ethical committee of the hospital, and the formal consent was not needed due to retrospective study (VNCH-RICH-18-96).

\subsection{Patient profiles}

TBV or so called double-outlet right ventricle with subpulmonary VSD, was defined as a heart with doubleoutlet right ventricle, sub-pulmonary VSD, without pulmonary stenosis, the Ao arises completely and the pulmonary artery (PA) more than 50\% from the right ventricle, the great arteries relationship maybe sideby-side or anteroposterior (10). We retrospective all patients diagnosed with TBV between June 2010 and December 2018, who underwent the ASO and VSD closure silmutaneously at National Children's Hospital, Hanoi, Vietnam. With the exception of our first patient, who underwent staged repair (coarctation repair without PA banding 2 weeks before the ASO), all of the remaining TBV with aortic arch anomalies successfully underwent single-stage total correction.

Exclusion criteria were the TBV patient who underwent ASO with PA banding and staged VSD closure.

\subsection{Surgical techniques}

The first patient underwent staged repair, with a thoracotomy approach for coarctation repair without PA banding, followed by the ASO performed via a median sternotomy approach later. All the remaining TBV patients in our study, including the patients who required aortic arch reconstruction as well, were treated via a single incision through a median sternotomy using isolated cerebral perfusion.

Standard bypass was established with an arterial cannula placed at the base of the innominate artery, bicaval cannulation and moderate hypothermia was used for all patients in our study. The autologous pericardium was harvested and kept in saline. The core temperature was decreased to $28^{\circ} \mathrm{C}$ during dissection, and near-infrared spectroscopy was used to monitor all patients. In cases in which coarctation or aortic arch reconstruction was required, the aortic cannula was advanced into the innominate artery, and antegrade cerebral perfusion was performed with the surgical technique for aortic arch reconstruction, as previously described (11). The closed technique for coronary transfer was used in all patients (12). We combined the trapdoor technique for left coronary button reimplantation and a simple incision for the right coronary button to minimize dilatation of the sinotubular junction and the sinus of Valsalva. The Lecompte maneuver was applied in all cases, irrespective of the relationship of the great arteries. The majority of patients underwent VSD closure through the right atrium using interrupted pledget sutures, except for 1 patient, who required an approach through the neopulmonary valve (neo-PV). For the upper part of the VSD, as the most difficult part of VSD closure through right atrial, a needle holder is used to shape the suture needle into a "fishhook" shape, and a forceps was used to temporary push down the outside of the pulmonary annulus. The surgeon can now easily see the superior aspect of the VSD, and close it through the tricuspid valve. The neo-PA 
was reconstructed using a fresh autologous pericardial patch, with particular care taken to trim the height and width of the patch to avoid torsion of the pulmonary bifurcation, which we considered to be the main cause of PA branch stenosis after the operation. We took meticulous care to make sure that the height of the neo-PA patch was equal to the height of the remaining anterior wall of the neo-PA, the width of the patch was not excessive and the diameter of the neo-PA after reconstruction was equal to the diameter of the PA bifurcation.

Before releasing the aortic cross clamp, the RVOT was always examined through the neo-PV. In cases where SPT were prominent and protruded into the RVOT, they were excised or divided totally from the RVOT up to the apex of the right ventricle, if possible, to avoid RVOTO. The key point to help surgeons realizes these structures and avoid resecting too much out of the heart is that these structures lie in an abnormal position, making connections adherent between the posterior ventricular septum at the infundibulum area superiorly, continuous with the tricuspid annulus at the area of inlet component of the right ventricle inferiorly, and the free wall of the right ventricle, which is not seen in the normal heart (Figure 1). These anomalous structures were described by Dr. Robert Anderson, which is also described as the right ventricular trabeculations arise from the anterior margin of the septomarginal trabeculation (page 41, Anatomy of the cardiac chambers) (13). These muscle bands are customary to the TBV lesion, are easily identified by RVOT inspection after ASO + VSD closure. Additionally, when the neo-PV annulus appeared hypoplastic, an approach through the tricuspid valve could be used to divide the SPTs.

The patent foramen ovale was stretched or a small atrial septal defect was left open to help decompress the right ventricle and maintain the cardiac output of the patient during intensive care management. Distal anastomosis of the neo-PA was completed after removal of the Ao cross clamp. In cases where the great arteries were side-by-side or the aorta arose from the anterior and right sides of the pulmonary system, the neo-PA was then shifted to the right PA to avoid coronary compression or torsion of the distal PA. The remaining defect in the PA bifurcation was closed by an autologous pericardial patch to avoid left PA stenosis.

In patients who required reoperation due to RVOTO, the procedure was performed through the neo-PA and the tricuspid valve, and the SPT remnants were resected, with the RV pressure being directly measured in combination with transesophageal echocardiography to confirm freedom from RVOTO. No transannular patches were required in our series for reoperation because we did not identify any cases of significant hypoplasticity of the neo-PV annulus during follow-up.

\subsection{Postoperative RVOT evaluation}

After discharge from the hospital, the patients were followed up at outpatient clinics in our hospital or the referring hospital with echocardiography, chest X-ray and electrocardiogram were regularly performed 1 month, 3 months, 6 months and annually, respectively. The patient's latest echocardiographic records were collected to evaluate the diameter and pressure gradient throughout the RVOT and neo-PV, as well as the continuous-wave Doppler measured tricuspid valve regurgitation velocity to estimate RV pressure.

The degree of RVOTO was evaluated according to EAE/ASE recommendations for the echocardiographic assessment of valve stenosis (14). The indication for reoperation was based on a peak RVOT doppler velocity and the clinical examination (Ross classification for heart failure in children): a peak RVOT measured in the distal RVOT greater than $35 \mathrm{mmHg}$ combined with Ross III assessment, or a peak ROVT greater than $50 \mathrm{mmHg}$ combined with Ross II assessment (15).

\subsection{Statistical analysis}

The normality of the variables was tested with the Shapiro-Wilk, and Skewness/Kurtosis tests. Continuous variables are expressed as the mean and standard deviation or median with the $25^{\text {th }}$ to $75^{\text {th }}$ percentile interval. Univariable analysis was applied with appropriate test (Fisher's exact tests or Chi squared tests), depending on the observed frequency. A paired t-test was used for the difference between two variables for the same subject. Possible risk factors for a poor outcome with $\mathrm{p}<0.25$ on Cox univariable analysis 
were assessed by competing risk analysis using the Stata software package (version 14.1; Stata Corp, Texas, USA). A p-value $<0.05$ was considered to indicate statistical significance. Actuarial survival, freedom from reoperation and reintervention were estimated by the Kaplan-Meier method.

\section{Results}

In the study period, there were 72 consecutive patients with median age on the day of operation was 58 days [interquartile range (IQR), 32.5 - 110.5], and the median weight was $3.75 \mathrm{~kg}$ (IQR, 3.2 - 4.5). Thirtyfive patients (48.6\%) underwent aortic arch reconstruction, which included 15 patients with coarctation, 16 patients with aortic arch hypoplasia, and 4 patients with aortic arch interruption. The details of the patient characteristics, surgical management and perioperative data are summarized in Tables 1 and 2.

\subsection{Risk factors for mortality}

Ten patients died in the hospital, and 2 patients died after discharge from the hospital during follow-up (8 and 10 months after the operation, respectively). The early mortality rate was $13.9 \%(10 / 72)$, and the overall mortality rate was $16.7 \%(12 / 72)$ at the median follow-up time of 48 months (9.25-73.75), with 10 patients lost to follow-up. Two patients in our series died within 24 hours postoperatively due to coronary malperfusion, 1 patient died iatrogenically due to the inotropic transfusion line being accidentally disconnected during patient transfer from the operating room to the ICU, and 1 patient died from intractable heart failure due to the misdiagnosis of left ventricular outflow tract obstruction caused by accessory mitral valve tissue. Additionally, 1 patient died on postoperative day (POD) \#5 because of uncontrollable arrhythmia (junctional ectopic tachycardia). The cause of death in the remaining 5 patients was related to nosocomial infection, with most of these patients requiring preoperative ventilation. The details of early mortality are described in Table 3.

There were two cases of late mortality. In the first case, the patient died suddenly at 8 months postoperatively, despite a recent echocardiogram showing normal biventricular function before death. The second case of late death occurred at 10 months after the operation; due to severe sepsis from pneumonia but had normal cardiac function at the latest follow-up. The estimated 1-year, 5-year, and 9-year freedom from death for the 72 patients were $83.2 \%(\mathrm{SD}=4.4), 83.2 \%(\mathrm{SD}=4.4)$ and $83.2 \%(\mathrm{SD}=4.4),(95 \% \mathrm{CI}, 0.7237$ to 0.9012$)$, respectively (Figure 2).

Competing risk analysis revealed that: intraoperative ventricular septal defect enlargement [hazard ratio (HR) 7.23, 95\% confidence interval (CI) 3.1294-16.7167; $P<0.001$ ], secondary aortic cross clamping (HR 28.38, 95\% CI 4.8427-166.3484; $P<0.001$ ), post-operative pneumonia (HR 5.64, 95\% CI 1.2724-24.9917; $P$ $=0.023$ ), and post-operative sepsis (HR 5.28, 95\% CI 1.3512-20.6553; $P=0.017$ ) were factors associated to overall mortality. By Cox multivariable analysis, the Ao arch hypoplasia (HR 2.96, 95\% CI 0.9170-9.5288; $P$ $=0.07)$ are approaching significant factors associated to overall mortality.

\subsection{Postoperative events and late reoperation}

Twenty-three patients required delayed sternal closure, almost all of whom were treated during 2010-2014. Postoperative arrhythmia occurred in 24 patients (33\%), including junctional ectopic tachycardia in 6 patients, supraventricular tachycardia in 3 patients, and sinus bradycardia in 15 patients, but no complete atrioventricular block. Nosocomial infection was one of the major complications in our series, with pneumonia in 17 patients, wound infection in 6 patients, sepsis in 5 patients, and a deep sternal wound infection in 1 patient.

There were 7 late reoperations in 5 patients, which included 5 reoperations for RVOTO in 3 patients during follow-up. One patient required pacemaker implantation due to sinus node dysfunction, and 1 patient developed ascending aorta stenosis after total correction for TBV with an interrupted aortic arch. One patient required balloon cardiac intervention for residual coarctation after the ASO with coarctation repair. Focusing on RVOTO, the median pressure gradient through the RVOT at the latest follow-up was $5 \mathrm{mmHg}$ (IQR, $3-9$ ). There were 2 patients with mild to moderate RVOTO (PG 28-35mmHg) only on echocardiography who may require reoperation in the future, but no symptoms were observed during exercise at the latest 
follow-up. No patients developed suprapulmonary stenosis or PA branch stenosis during follow-up. The latest echocardiogram revealed no or trivial neo-aortic valve regurgitation in 48 patients, and 2 patients had mild aortic regurgitation. The estimated freedom from RVOTO reoperation for this cohort at 1 year, 5 years and 9 years was $98.3 \%(\mathrm{SD}=1.7)(95 \% \mathrm{CI}, 0.8857$ to 0.9976$), 91.9 \%(\mathrm{SD}=4.8)(95 \% \mathrm{CI}, 0.7524$ to 0.9749$)$ and $91.9(\mathrm{SD}=4.8)$ (95\% CI, 0.7524 to 0.9749 ), respectively (Figure 3).

Concerning the possibility of neo-PV growth, the diameter and the Z-score of the neo-PV in the subgroup of TBV associated with aortic arch anomalies was calculated preoperatively and at the last follow-up on echocardiography and showed significant growth $(P<0.001$ and $P=0.001$, respectively), as shown in Figure 4.

\section{Discussion}

Recently, the results of the ASO for TBV have been excellent, with low hospital mortality rates as reported by Vergnat et al. (16) (5.8\%), Soszyn et al. (6) (5.3\%), and Sinzobahamvya et al. (7) (2.9\%), among others. The risk factors for overall mortality are related to the coronary patterns, a higher weight at the time of the operation, preoperative PA banding, aortic arch anomaly and postoperative subneopulmonary obstruction $(1,6,16,17)$. Results from our study, which were achieved in LMIC show that, intraoperative ventricular septal defect enlargement $[\mathrm{HR}=7.23, P<0.001]$, secondary aortic cross clamping (HR 28.38, $P<0.001$ ), post-operative pneumonia (HR 5.64, $P=0.023$ ), and post-operative sepsis (HR 5.28, $P=0.017$ ) were factors associated to overall mortality. These results reflect the reality of the learning curve faced by a congenital heart program in a LMIC as they develop their management of complex lesions, including dealing with missed diagnosis, late presentation and nosocomial infection.

According to many previous reports, TBV is a risk factor for RVOTO-related reoperation after the ASO compared to simple transposition of the great arteries (TGA) or even complex TGA $(7,8,18,19)$. Realizing this problem, we attempted to avoid RVOTO as much as possible by systematically checking the RVOT and totally resecting or dividing SPTs inferiorly up to the apex of the right ventricle (14). Interestingly, in 3 patients who required reoperation for RVOTO in our study, 2 of them did not undergo the RVOT procedure to resect SPTs during the ASO, as their procedures were performed in the early period of the study. The remaining patient who developed RVOTO was a TBV patient with type B aortic arch interruption, and her symptoms occurred 6 months after the ASO despite the attempt to resect the SPTs in the first operation. During the reoperation, the level of the RVOTO was far from the neo-PV, and we performed resection of the SPTs through the neo-PV and the tricuspid valve. We also noted that the right ventricle end diastolic volume may increase after SPT resection as the free wall of the right ventricle is now free from the ventricular septum.

Risk for RVOTO has been reported to occur in up to $53 \%$ of patients after ASO with TBV and remains a major cause of late morbidity ( 7 ) . Even when a preventative strategy is applied, reintervention due to RVOTO remains as high (20-35\%). Only the experience from the Royal Melbourne Children's Hospital reported an RVOT reintervention rate of less than $10 \%$, specifically $2.2 \%$ (6). So, our rate of RVOT reintervention of only $6 \%$, with a larger cohort is noteworthy, and the estimated freedom for RVOTO reoperation was $91.9 \%(\mathrm{SD}=4.8)(95 \% \mathrm{CI}, 0.7524$ to 0.9749$)$ at 9 years follow-up. A smaller native aortic valve annulus has been identified as a risk factor for RVOT reintervention (8). In contrast, we demonstrated growth of the neo-PV annulus as shown in the Fig. 4. We attribute this RVOT growth and the low incidence of RVOTO reintervention to our policy of aggressive division and/or resection of SPT.

Another common reason for reoperation after the ASO is supravalvular neo-PA stenosis (1-3, 17). However, in our series, we did not have any patients who developed neo-PA stenosis requiring either reoperation or reintervention. This complication was also rare in our ASO cohort (1/445 ASO patients). Attempts were made to size the autologous pericardial patch to be equal the height of the neo-PA, while the width of the neo-PA was equal to the diameter of the pulmonary bifurcation. We believe that a large patch does not guarantee the normal growth of the PA in the long term; instead, a size difference between the neopulmonary and pulmonary bifurcation may create turbulent flow at the anastomotic site and easily lead to stenosis. 
This approach has helped us avoid torsion of the neo-PA bifurcation and has allowed for normal growth of the neo-PA.

Limitations

This was a retrospective study, and additional long-term follow-up is needed to confirm the results. Complete patient data follow up was approximately $84 \%$; bias and the relatively incomplete follow-up dataset might limit the validity of our results.

Due to the limitation of a rare congenital heart disease, the small number of events might influence to the power of statistical analysis. In condition of a lower-middle income country, the post-operative sepsis and post-operative pneumonia certainly are predictors for hospital mortality, but all risk factors (included intraoperative ventricular septal defect enlargement and secondary aortic cross clamping) are interrelated.

\section{Conclusions}

The surgical results of TBV were satisfactory in the operative setting of a LMIC with limited resources. Understanding the anatomical structure of the RVOT in TBV is crucial for pediatric cardiovascular surgeons, and performing extensive SPT division or resection may reduce the reintervention rate for RVOTO after the ASO in TBV patients.

Conflict of interest: The author(s) declared no potential conflicts of interest with respect to the research, authorship, and/or publication of this article.

\section{Author contributions}

Truong Nguyen Ly Thinh (Conceptualization; Project administration; Investigation; Visualization; Writing - review \& editing)

Vinh Tran Quang (Data curation; Formal analysis; review \& editing)

Mai Nguyen Tuan (Writing - review \& editing)

Duyen Mai Dinh (Writing - review \& editing)

Anh Doan Vuong (Formal analysis; Writing - review \& editing)

Data sharing: The authors confirm that the data supporting the findings of this study are available from the corresponding author on request.

Reference

1. Griselli M, McGuirk SP, Ko C-S, Clarke AJB, Barron DJ, Brawn WJ. Arterial switch operation in patients with Taussig-Bing anomaly - influence of staged repair and coronary anatomy on outcome. European Journal of Cardio-Thoracic Surgery. 2007 Feb;31(2):229-35.

2. Hayes DA, Jones S, Quaegebeur JM, Richmond ME, Andrews HF, Glickstein JS, et al. Primary Arterial Switch Operation as a Strategy for Total Correction of Taussig-Bing Anomaly: A 21-Year Experience. Circulation. 2013 Sep 10;128(11_suppl_1):S194-8.

3. Alsoufi B, Cai S, Williams WG, Coles JG, Caldarone CA, Redington AM, et al. Improved results with single-stage total correction of Taussig-Bing anomaly. European Journal of Cardio-Thoracic Surgery. 2008 Feb;33(2):244-50.

4. Luo K, Zheng J, Wang S, Zhu Z, Gao B, Xu Z, et al. Single-Stage Correction for Taussig-Bing Anomaly Associated With Aortic Arch Obstruction. Pediatr Cardiol. 2017 Dec 1;38(8):1548-55.

5. Comas J, Mignosa C, Cochrane A, Wilkinson J, Karl T. Taussig-Bing anomaly and arterial switch: aortic arch obstruction does not influence outcome. European Journal of Cardio-Thoracic Surgery. 1996;10(12):1114-9. 
6. Soszyn N, Fricke TA, Wheaton GR, Ramsay JM, d'Udekem Y, Brizard CP, et al. Outcomes of the Arterial Switch Operation in Patients With Taussig-Bing Anomaly. The Annals of Thoracic Surgery. 2011 Aug;92(2):673-9.

7. Sinzobahamvya N, Blaschczok HC, Asfour B, Arenz C, Jussli MJ, Schindler E, et al. Right ventricular outflow tract obstruction after arterial switch operation for the Taussig-Bing heart. European Journal of Cardio-Thoracic Surgery. 2007 May;31(5):873-8.

8. Bokenkamp R, Aguilar E, van der Palen RLF, Sojak V, Bruggemans EF, Hruda J, et al. Reoperation for right ventricular outflow tract obstruction after arterial switch operation for transposition of the great arteries and aortic arch obstruction. Eur J Cardiothorac Surg. 2016 May;49(5):e91-6.

9. Williams WG, Quaegebeur JM, Kirklin JW, Blackstone EH. Outflow obstruction after the arterial switch operation: A multiinstitutional study. The Journal of Thoracic and Cardiovascular Surgery. 1997 Dec;114(6):975-90.

10. Walters HL, Mavroudis C, Tchervenkov CI, Jacobs JP, Lacour-Gayet F, Jacobs ML. Congenital Heart Surgery Nomenclature and Database Project: double outlet right ventricle. The Annals of Thoracic Surgery. 2000 Mar;69(3):249-63.

11. Truong NLT, Mai NT, Vinh TQ, Anh DV, Duyen MD. Single-stage repair for coarctation with ventricular septal defect: results of 100 cases at a single centre. Interactive CardioVascular and Thoracic Surgery. 2020 Oct 1;31(4):559-64.

12. Bove EL. Current Technique of the Arterial Switch Procedure for Transposition of the Great Arteries. J Cardiac Surgery. 1989 Sep;4(3):193-9.

13. Anderson RH, Spicer DE, Hlavacek AM, Cook AC, Backer CL. Wilcox's Surgical Anatomy of the Heart [Internet]. 2013 [cited 2020 Oct 11]. Available from: http://dx.doi.org/10.1017/CBO9781139028561

14. Baumgartner H, Hung J, Bermejo J, Chambers JB, Evangelista A, Griffin BP, et al. Echocardiographic assessment of valve stenosis: EAE/ASE recommendations for clinical practice. European Journal of Echocardiography. 2009 Jan 1;10(1):1-25.

15. Ross RD. The Ross Classification for Heart Failure in Children After 25 Years: A Review and an Age-Stratified Revision. Pediatr Cardiol. 2012 Dec;33(8):1295-300.

16. Vergnat M, Baruteau A-E, Houyel L, Ly M, Roussin R, Capderou A, et al. Late outcomes after arterial switch operation for Taussig-Bing anomaly. The Journal of Thoracic and Cardiovascular Surgery. 2015 Apr;149(4):1124-32.

17. Urban A, Brecher A. The Arterial Switch Repair and the Obstructive Right Ventricular Outflow Tract: Does It Matter? Thorac cardiovasc Surg. 1991 Aug;39(S 2):170-5.

18. Daebritz SH, Nollert G, Sachweh JS, Engelhardt W, von Bernuth G, Messmer BJ. Anatomical risk factors for mortality and cardiac morbidity after arterial switch operation. The Annals of Thoracic Surgery. 2000 Jun;69(6):1880-6.

19. Wetter J, Sinzobahamvya N, Blaschczok HC, Cho M-Y, Brecher AM, Gravinghoff LM, et al. Results of arterial switch operation for primary total correction of the Taussig-Bing anomaly. The Annals of Thoracic Surgery. 2004 Jan;77(1):41-6.

Tables

Table 1. Demographic and anatomical characteristics $(\mathrm{n}=72)$

\begin{tabular}{ll}
\hline Patient characteristics & $\mathrm{n}(\%)$ or median (IR) \\
\hline Male & $56(78)$
\end{tabular}




\begin{tabular}{ll}
\hline Patient characteristics & $\mathrm{n}(\%)$ or median (IR) \\
\hline Age (days) & $58(32.5-110.5)$ \\
Weight (kg) & $3.7(3.2-4.5)$ \\
Preoperative ventilation & $13(18)$ \\
Balloon atrial septostomy & $3(4)$ \\
Preoperative infection & $4(6)$ \\
Aortic arch obstruction & $35(49)$ \\
Aortic arch hypoplasia and coarctation of the aorta & $31(43)$ \\
Interrupted aortic arch & $4(6)$ \\
Coronary anatomy (Leiden convention) & \\
1LCx-2R & $22(31)$ \\
1L-2RCx & $19(26)$ \\
1R-2LCx & $8(11)$ \\
1LR-2Cx & $5(7)$ \\
2RLCx & $14(19)$ \\
1LCxR & $4(6)$ \\
Intramural coronary course & $5(7)$ \\
Ao-PA relationship & \\
Side-by-side & $29(40)$ \\
Ao anterior and rightward & $14(20)$ \\
Ao directly anterior & $29(40)$ \\
Native PA-Ao diameter ratio (ratio [?] 2) & $63(88)$ \\
Commissure malalignment & $18(25)$ \\
Additional cardiac anomalies & \\
m-VSD & $3(4)$ \\
Mitral valve abnormality & $2(3)$ \\
ASD & $26(36)$ \\
Bicuspid native PV & $2(3)$ \\
\hline &
\end{tabular}

Ao: aorta; ASD: atrial septal defect; m-VSD: muscular ventricular septal defect; PA: pulmonary artery; PV: pulmonary valve

Table 2. Operative and early postoperative data

\begin{tabular}{ll}
\hline Characteristics & $\mathrm{n}(\%)$, mean $( \pm \mathrm{SD})$, or median (IR) \\
\hline Operative times & $166 \mathrm{~min}(37)$ \\
Aortic cross-clamping time & $249 \mathrm{~min}(94)$ \\
Bypass time & $33 \mathrm{~min}(13)$ \\
Regional cerebral perfusion time & $35(49)$ \\
Additional procedures & $2(3)$ \\
Aortic arch reconstruction & $2(3)$ \\
VSD enlargement & $60(83)$ \\
Mitral valvuloplasty & $30(42)$ \\
SPT resection or division & $37(51)$ \\
Pulmonary bifurcation translocation (to the right PA) & $10(14)$ \\
Resizing of the neo-Ao & \\
Hospital mortality & $4(6)$ \\
Complications & $23(32)$ \\
Intraoperative re-exploration &
\end{tabular}




\begin{tabular}{ll}
\hline Characteristics & $\mathrm{n}(\%)$, mean $( \pm \mathrm{SD})$, or median $(\mathrm{IR})$ \\
\hline Arrhythmia & $24(33)$ \\
Wound infection & $6(8)$ \\
Respiratory infection & $17(24)$ \\
Sepsis & $5(7)$ \\
Phrenic nerve palsy & $1(1)$ \\
Neurological abnormality or seizures & $2(3)$ \\
CAB requiring permanent pacemaker & 0 \\
\hline
\end{tabular}

CAB: complete atrioventricular block; PA: pulmonary artery; SPT: septoparietal trabeculations; VSD: ventricular septal defect.

Table 3. Cause of death

\begin{tabular}{|c|c|c|c|c|}
\hline Patient & Diagnosis & Procedure & Time of death & Cause of death \\
\hline & $\begin{array}{l}\text { TBV-CoA-AAH- } \\
\text { LVOTO }\end{array}$ & $\begin{array}{l}\text { ASO + VSD closure } \\
+ \text { aortic arch } \\
\text { reconstruction }+ \\
\text { secondary aortic } \\
\text { cross clamping to } \\
\text { resect accessory MV } \\
\text { tissue }\end{array}$ & POD \#1 (6 hours) & $\begin{array}{l}\text { LCOS (undetected } \\
\text { LVOTO due to } \\
\text { accessory MV } \\
\text { tissue) }\end{array}$ \\
\hline & TBV CoA AAH & $\begin{array}{l}\text { ASO + VSD closure } \\
+ \text { aortic arch } \\
\text { reconstruction }\end{array}$ & POD \#11 & $\begin{array}{l}\text { Respiratory } \\
\text { infection } \\
\text { (preoperative } \\
\text { ventilation) }\end{array}$ \\
\hline & TBV CoA & $\begin{array}{l}\mathrm{ASO}+\mathrm{VSD} \text { closure } \\
+ \text { coarctation repair }\end{array}$ & POD \#1 (12 hours) & $\begin{array}{l}\text { Cardiac arrest, } \\
\text { myocardial } \\
\text { malperfusion }\end{array}$ \\
\hline & TBV CoA & $\begin{array}{l}\text { ASO + VSD closure } \\
+ \text { coarctation repair }\end{array}$ & POD \#28 & $\begin{array}{l}\text { Respiratory } \\
\text { infection }\end{array}$ \\
\hline & TBV CoA & $\begin{array}{l}\text { ASO + VSD closure } \\
+ \text { coarctation repair }\end{array}$ & POD \#6 & $\begin{array}{l}\text { Respiratory } \\
\text { infection } \\
\text { (preoperative } \\
\text { ventilation) }\end{array}$ \\
\hline & TBV CoA AAH & $\begin{array}{l}\text { ASO + VSD closure } \\
+ \text { aortic arch } \\
\text { reconstruction }\end{array}$ & POD \#4 & $\begin{array}{l}\text { Respiratory } \\
\text { infection } \\
\text { (preoperative } \\
\text { ventilation) }\end{array}$ \\
\hline & TBV IAA type A & $\begin{array}{l}\text { ASO + VSD closure } \\
+ \text { aortic arch } \\
\text { reconstruction }\end{array}$ & POD \#40 & $\begin{array}{l}\text { Sepsis, severe TV, } \\
\text { chylothorax } \\
\text { (preoperative } \\
\text { ventilation) }\end{array}$ \\
\hline & TBV & $\mathrm{ASO}+\mathrm{VSD}$ closure & $\mathrm{POD} \# 1$ (in $\mathrm{OR}$ ) & $\begin{array}{l}\text { LCOS (undetected } \\
\text { disruption of } \\
\text { inotropic infusion } \\
\text { line) }\end{array}$ \\
\hline & TBV & ASO + VSD closure & POD \#5 & $\begin{array}{l}\text { Intractable heart } \\
\text { failure (junctional } \\
\text { ectopic tachycardia) }\end{array}$ \\
\hline
\end{tabular}




\begin{tabular}{|c|c|c|c|c|}
\hline Patient & Diagnosis & Procedure & Time of death & Cause of death \\
\hline & TBV & $\begin{array}{l}\text { ASO + VSD closure } \\
+ \text { secondary aortic } \\
\text { cross clamping due } \\
\text { to coronary } \\
\text { malperfusion }\end{array}$ & POD \#1 (4 hours) & $\begin{array}{l}\text { Cardiac arrest, } \\
\text { myocardial } \\
\text { malperfusion }\end{array}$ \\
\hline
\end{tabular}

AAH: aortic arch hypoplasia; ASO: arterial switch operation; CoA: coarctation; IAA: interrupted aortic arch; LVOTO: left ventricular outflow tract obstruction; LCOS: low cardiac output syndrome; MV: mitral valve; POD: postoperative day; OR: operating room; TV: tricuspid valve; VSD closure: ventricular septal defect closure.

\section{Figure legends}

Figure 1. Position of SPTs during the operation and how to avoid RVOTO after the ASO in TBV patients (VS: ventricular septum; SPTs: septoparietal trabeculations; RV: right ventricle)

Figure 2. Kaplan-Meier curve showing the survival of 72 patients with TBV after the ASO

Figure 3. Kaplan-Meier curve showing freedom from reintervention for 72 patients with TBV after the ASO

Figure 4. Nomogram showing the significant difference in the diameter and the Z-score of the neopulmonary annulus before and after the ASO in TBV patients with aortic arch anomalies $(\mathrm{n}=28 ; P<0.000$ and $P=$ 0.001 , respectively)

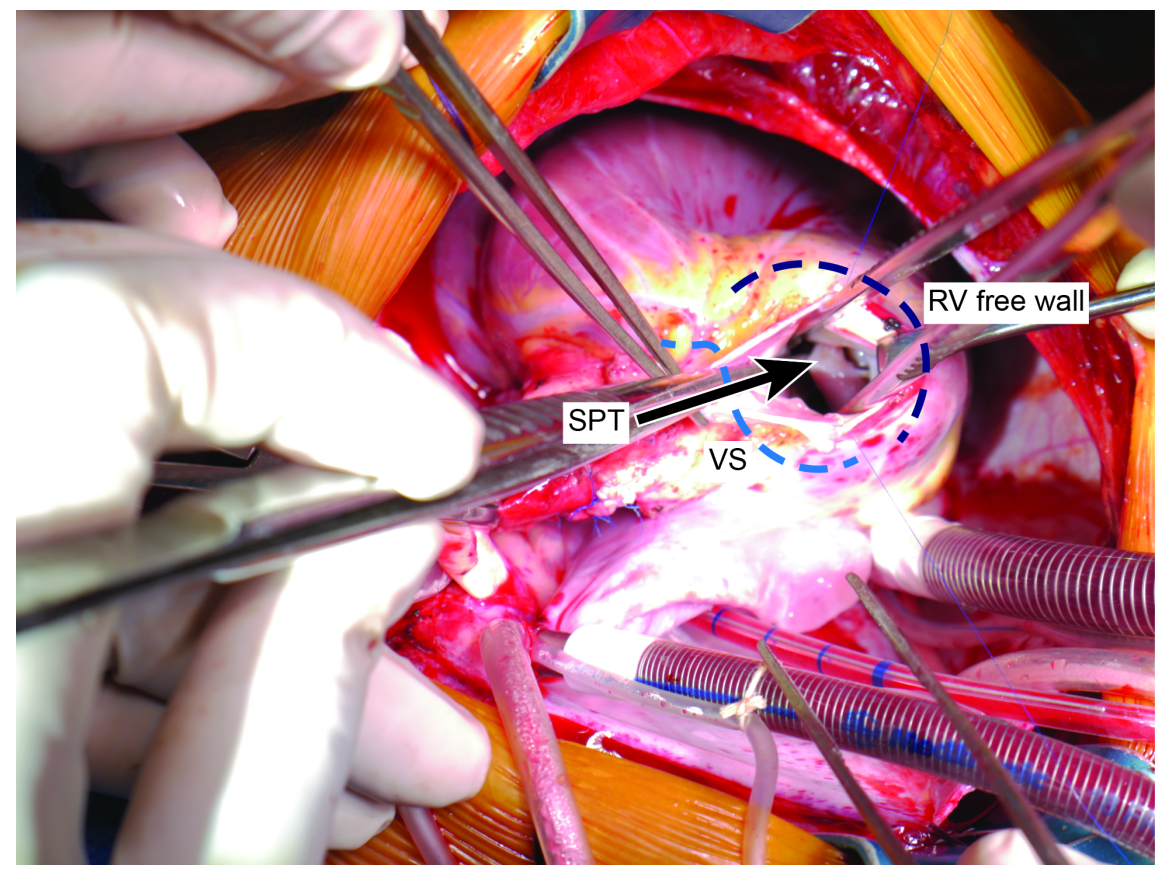



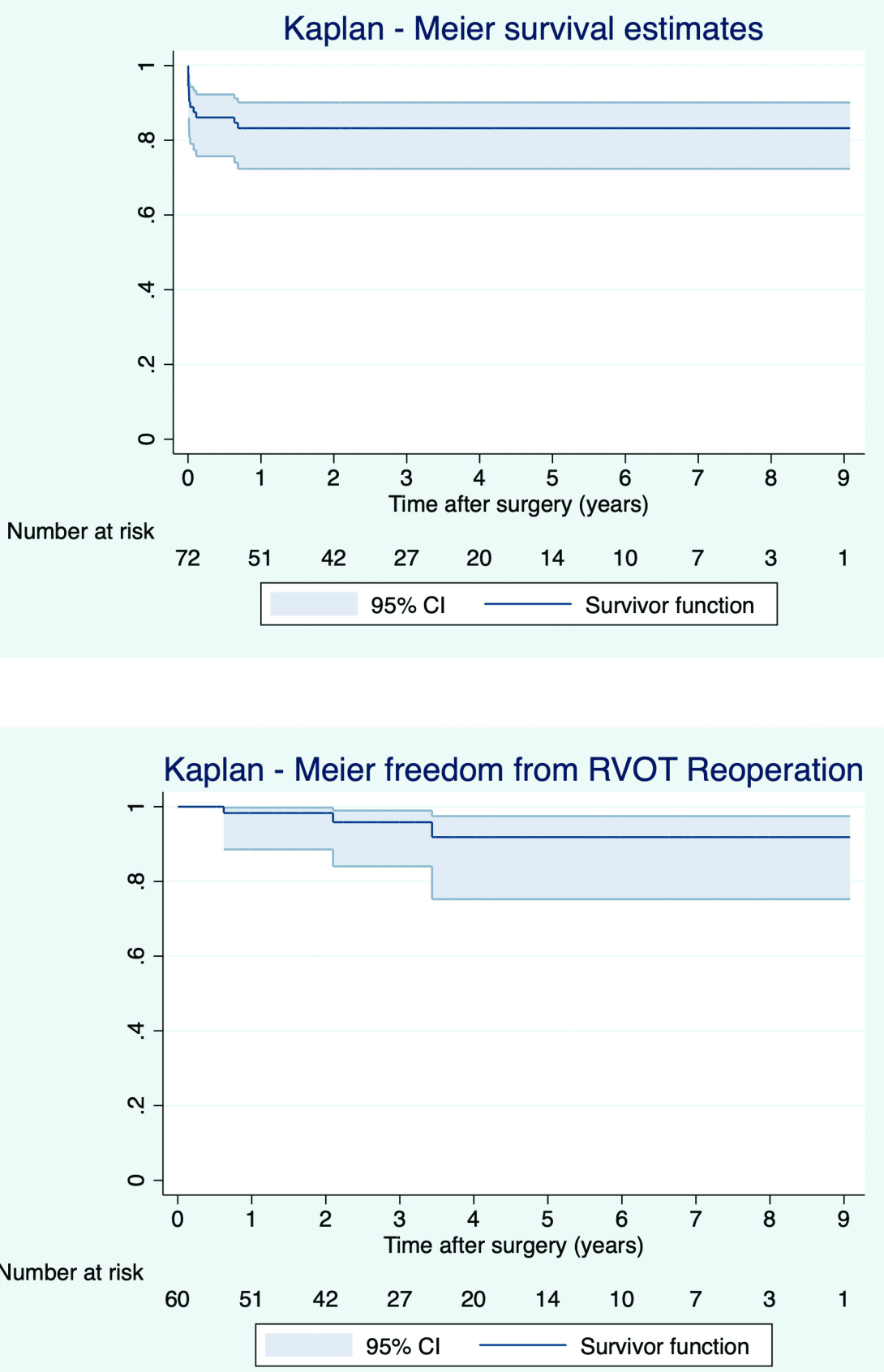


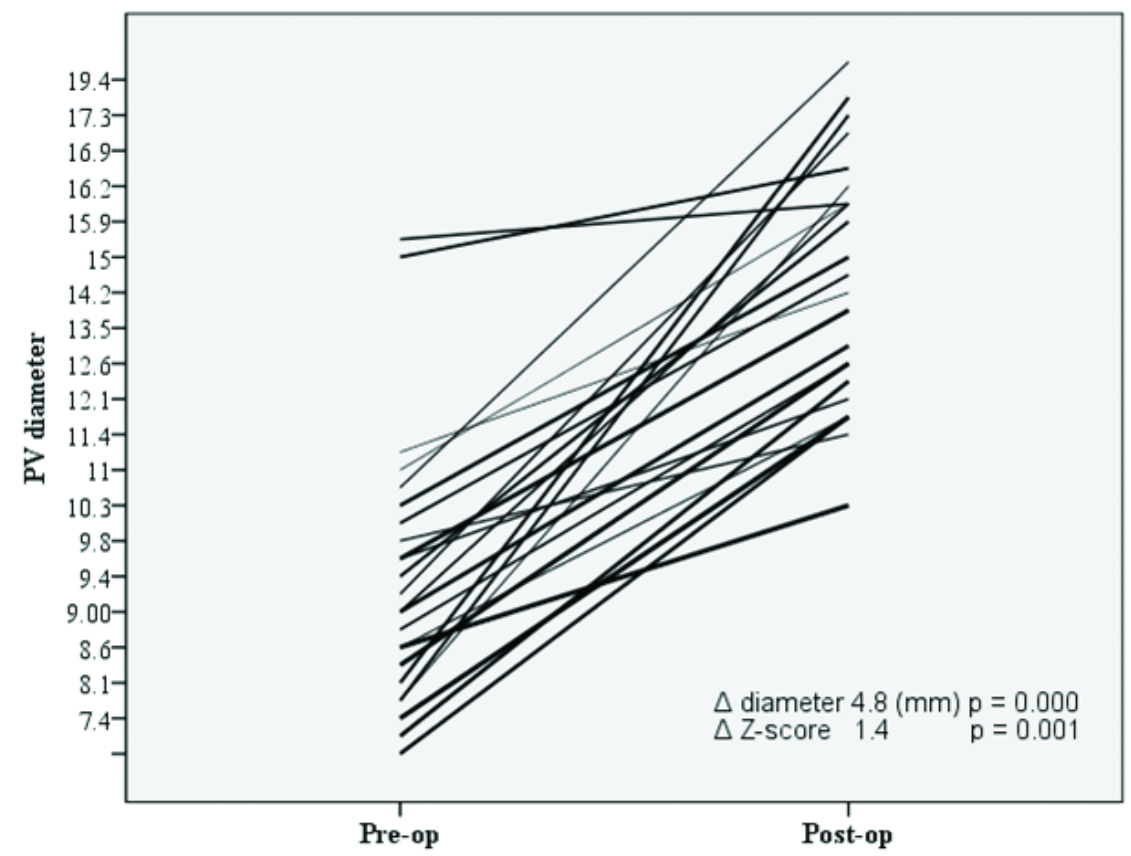

